\begin{tabular}{|c|c|}
\hline Title & Critical Modules of the Ring of Differential Operators of an A ffine Semigroup A Igebra \\
\hline Author(s) & Saito, Mutsumi \\
\hline Citation & $\begin{array}{l}\text { Communications in A Igebra, 38(2), 618-631 } \\
\text { https://doi.org/10.1080/00927870902828603 }\end{array}$ \\
\hline Issue Date & $2010-02$ \\
\hline DOC URL & http:/hdl.handle.net/2115/44781 \\
\hline Rights & $\begin{array}{l}\text { This is an electronic version of an article published in Communications in A lgebra, 38(2), Feb. 2010, 618-631. } \\
\text { Communications in A lgebra is available online at: http://www.informaworld.com/openurl ?genre=article\&issn=0092- } \\
7872 \& \text { volume }=38 \& \text { issue }=2 \& \text { spage }=618\end{array}$ \\
\hline Type & article (author version) \\
\hline File Information & CiA 38-2_618-631.pdf \\
\hline
\end{tabular}

Instructions for use 


\title{
CRITICAL MODULES \\ OF THE RING OF DIFFERENTIAL OPERATORS OF AN AFFINE SEMIGROUP ALGEBRA
}

\author{
MUTSUMI SAITO
}

\begin{abstract}
Let $D$ be the ring of differential operators of an affine semigroup algebra. Regarding the Krull dimension of finitely generated $\mathbb{Z}^{d}$-graded $D$-modules, we characterize critical $\mathbb{Z}^{d}$-graded $D$-modules. Moreover we explicitly describe cyclic ones.
\end{abstract}

Mathematics Subject Classification (2000): Primary 13N10; Secondary 16S32.

Keywords: Critical modules, rings of differential operators, affine semigroup algebras

\section{INTRODUCTION}

Critical modules were introduced by Hart (1971) and Goldie (1972). They are the modules whose quotient by any nonzero submodule has strictly smaller Krull dimension (in the sense of (Gabriel, 1962) and (Rentschler, Gabriel, 1967)). The critical modules of Krull dimension 0 are precisely the simple modules. The notions of Krull dimension and critical modules enable us to use Artinian type method to Noetherian rings (see e.g. (Goodearl, Warfield, 1989; Lenagan, 2000; McConnel, Robson, 1987)).

Let $F$ be an algebraically closed field of characteristic 0 . Let $A:=\left\{\boldsymbol{a}_{1}, \boldsymbol{a}_{2}, \ldots, \boldsymbol{a}_{n}\right\}$ be a finite subset of $\mathbb{Z}^{d}$. Let $R_{A}$ denote the semigroup algebra over $F$ of the monoid generated by $A$, and $D\left(R_{A}\right)$ the ring of differential operators of $R_{A}$; both of them have natural $\mathbb{Z}^{d_{-}}$ gradings. Musson, Van den Bergh (1998) classified the $\mathbb{Z}^{d}$-graded simple $D\left(R_{A}\right)$-modules. (See also (Saito, Traves, 2001).) In this paper, considering the Krull dimension of finitely generated $\mathbb{Z}^{d}$-graded $D\left(R_{A}\right)$-modules, we characterize critical $\mathbb{Z}^{d}$-graded $D\left(R_{A}\right)$-modules. Moreover we explicitly describe cyclic critical $\mathbb{Z}^{d}$-graded $D\left(R_{A}\right)$-modules.

To consider the Krull dimension, it is convenient to assume the Noetherian property. (Saito, Takahashi, 2008) proved that $D\left(R_{A}\right)$ is always right Noetherian. It also gave some conditions for the left Noetherian property; for example, $D\left(R_{A}\right)$ is left Noetherian if $R_{A}$ satisfies Serre's $\left(S_{2}\right)$ condition. Throughout this paper, we assume that $D\left(R_{A}\right)$ is left Noetherian when we consider left $D\left(R_{A}\right)$-modules.

This paper is organized as follows. In Section 2, we recall some fundamental facts about the rings of differential operators of affine semigroup algebras.

Let $\boldsymbol{a}, \boldsymbol{b} \in F^{d}$. In (Saito, Traves, 2001), a preorder $\boldsymbol{a} \preceq \boldsymbol{b}$ was defined; the equivalence relation induced from $\preceq$ was used for the classifications of $\mathbb{Z}^{d}$-graded simple modules and $A$-hypergeometric systems (Musson, Van den Bergh, 1998; Saito, 2001; Saito, Traves, 2001; Saito, 2007). This preorder is for maximal ideals of the polynomial ring $F\left[s_{1}, \ldots, s_{d}\right]$. In Section 3, we generalize this for prime ideals, suitable for considering critical modules.

In Section 4 , we introduce $\widetilde{\mathcal{O}}$ (resp. ${ }^{R} \widetilde{\mathcal{O}}$ ), the category of $\mathbb{Z}^{d}$-graded finitely generated left (resp. right) $D\left(R_{A}\right)$-modules. For an object $M=\bigoplus_{\boldsymbol{a} \in \mathbb{Z}^{d}} M_{\boldsymbol{a}} \in \widetilde{\mathcal{O}}$ (resp. ${ }^{R} \widetilde{\mathcal{O}}$ ), we 
define $\delta(M)$ by

$$
\delta(M):=\max _{\boldsymbol{a}} \operatorname{dim}_{F[s]} M_{\boldsymbol{a}} .
$$

In Section 5 , we define $\operatorname{deg}(M)$ for $M \in \widetilde{\mathcal{O}}$ (resp. ${ }^{R} \widetilde{\mathcal{O}}$ ) so that, for a descending chain

$$
M=M_{0} \supseteq M_{1} \supseteq M_{2} \supseteq \cdots \supseteq M_{i} \supseteq \cdots,
$$

there exist at most $\operatorname{deg}(M) i$ 's such that $\delta\left(M_{i} / M_{i+1}\right)=\delta(M)$.

In Section 6, we prove that $\delta$ gives the Krull dimension for the category $\widetilde{\mathcal{O}}$ (resp. ${ }^{R} \widetilde{\mathcal{O}}$ ).

In Section 7 , we characterize critical $\mathbb{Z}^{d}$-graded $D\left(R_{A}\right)$-modules (Theorem 7.4), and explicitly describe cyclic ones (Theorem 7.7).

\section{Rings of Differential operators of AFFine SEMigroup ALGEBRAS}

In this section, we briefly recall some fundamental facts about the rings of differential operators of affine semigroup algebras. Let $F$ be an algebraically closed field of characteristic 0 . Let $A:=\left\{\boldsymbol{a}_{1}, \boldsymbol{a}_{2}, \ldots, \boldsymbol{a}_{n}\right\}$ be a finite set of column vectors in $\mathbb{Z}^{d}$, and $\mathbb{N} A$ the monoid generated by $A$. Throughout this paper, we assume that $\mathbb{Z}^{d}$ is generated by $A$ as an abelian group for simplicity.

The ring $F\left[t_{1}^{ \pm 1}, \ldots, t_{d}^{ \pm 1}\right]\left\langle\partial_{1}, \ldots, \partial_{d}\right\rangle$ of differential operators with Laurent polynomial coefficients is the ring of differential operators on the algebraic torus $\left(F^{\times}\right)^{d}$, where $\left[\partial_{i}, t_{j}\right]=$ $\delta_{i j},\left[\partial_{i}, t_{j}^{-1}\right]=-\delta_{i j} t_{j}^{-2}$, and the other pairs of generators commute. Here $[$,$] denotes the$ commutator, and $\delta_{i j}$ is 1 if $i=j$ and 0 otherwise.

The semigroup algebra $R_{A}:=F[\mathbb{N} A]=\bigoplus_{\boldsymbol{a} \in \mathbb{N} A} F t^{\boldsymbol{a}}$ is the ring of regular functions on the affine toric variety defined by $A$, where $t^{\boldsymbol{a}}=t_{1}^{a_{1}} t_{2}^{a_{2}} \cdots t_{d}^{a_{d}}$ for $\boldsymbol{a}={ }^{t}\left(a_{1}, a_{2}, \ldots, a_{d}\right)$. Its ring of differential operators $D\left(R_{A}\right)$ can be realized as a subring of the ring

$$
F\left[t_{1}^{ \pm 1}, \ldots, t_{d}^{ \pm 1}\right]\left\langle\partial_{1}, \ldots, \partial_{d}\right\rangle
$$

of differential operators on the big torus as follows:

$$
D\left(R_{A}\right)=\left\{P \in F\left[t_{1}^{ \pm 1}, \ldots, t_{d}^{ \pm 1}\right]\left\langle\partial_{1}, \ldots, \partial_{d}\right\rangle: P\left(R_{A}\right) \subset R_{A}\right\} .
$$

Put $s_{j}:=t_{j} \partial_{j}$ for $j=1,2, \ldots, d$. Then it is easy to see that $s_{j} \in D\left(R_{A}\right)$ for all $j$. We introduce a $\mathbb{Z}^{d}$-grading on the ring $D\left(R_{A}\right)$ as follows: For $\boldsymbol{a}={ }^{t}\left(a_{1}, a_{2}, \ldots, a_{d}\right) \in \mathbb{Z}^{d}$, set

$$
D\left(R_{A}\right)_{\boldsymbol{a}}:=\left\{P \in D\left(R_{A}\right):\left[s_{j}, P\right]=a_{j} P \quad \text { for } j=1,2, \ldots, d\right\} .
$$

Then $D\left(R_{A}\right)=\bigoplus_{\boldsymbol{a} \in \mathbb{Z}^{d}} D\left(R_{A}\right)_{\boldsymbol{a}}$.

By regarding $D\left(R_{A}\right)_{\boldsymbol{a}}$ as a subset of $F\left[t_{1}^{ \pm 1}, \ldots, t_{d}^{ \pm 1}\right]\left\langle\partial_{1}, \ldots, \partial_{d}\right\rangle$, we see that there exists an ideal $I$ of $F[s]:=F\left[s_{1}, \ldots, s_{d}\right]$ such that $D\left(R_{A}\right)_{\boldsymbol{a}}=t^{\boldsymbol{a}} I$. To describe this ideal $I$ explicitly, we define a subset $\Omega(\boldsymbol{a})$ of the semigroup $\mathbb{N} A$ by

$$
\Omega(\boldsymbol{a})=\{\boldsymbol{b} \in \mathbb{N} A: \boldsymbol{b}+\boldsymbol{a} \notin \mathbb{N} A\}=\mathbb{N} A \backslash(-\boldsymbol{a}+\mathbb{N} A) .
$$

Then each $D\left(R_{A}\right)_{\boldsymbol{a}}$ is described as follows.

Theorem 2.1 (Theorem 2.3 in (Musson, 1987)).

$$
D\left(R_{A}\right)_{\boldsymbol{a}}=t^{\boldsymbol{a}} \mathbb{I}(\Omega(\boldsymbol{a})) \quad \text { for all } \boldsymbol{a} \in \mathbb{Z}^{d},
$$

where

$$
\mathbb{I}(\Omega(\boldsymbol{a})):=\left\{f(s) \in F[s]:=F\left[s_{1}, \ldots, s_{d}\right]: f \text { vanishes on } \Omega(\boldsymbol{a})\right\}
$$


In particular, we have for each $\boldsymbol{a} \in \mathbb{N} A$

$$
D\left(R_{A}\right)_{\boldsymbol{a}}=t^{\boldsymbol{a}} F[s]=F[s] t^{\boldsymbol{a}},
$$

since $\Omega(\boldsymbol{a})=\emptyset$ in this case.

\section{EQUivalenCE RELATIONS}

In this section, for a prime ideal $\mathfrak{p}$ of $F[s]$, we define a preorder $\preceq_{[\mathfrak{p}]}$ and an equivalence relation $\sim_{[\mathfrak{p}]}$, which are generalizations of those considered in (Musson, Van den Bergh, 1998; Saito, 2001; Saito, Traves, 2001; Saito, 2007) for maximal ideals (Proposition 3.3 and Remark 3.4). This equivalence relation plays a central role in this paper.

Notation. For an ideal $I$ of $F[s]$ and $\boldsymbol{c} \in \mathbb{Z}^{d}$, we define a new ideal $I+\boldsymbol{c}$ by

$$
I+c:=\{f(s-c): f(s) \in I\} .
$$

We clearly have the following.

\section{Proposition 3.1. (1) $\mathbb{I}(V)+\boldsymbol{c}=\mathbb{I}(V+\boldsymbol{c})$.}

(2) $\mathfrak{m}_{\boldsymbol{a}}+(\boldsymbol{b}-\boldsymbol{a})=\mathfrak{m}_{\boldsymbol{b}}$, where $\mathfrak{m}_{\boldsymbol{a}}$ is the maximal ideal $\left\langle s_{1}-a_{1}, \ldots, s_{d}-a_{d}\right\rangle$ for $\boldsymbol{a}={ }^{t}\left(a_{1}, \ldots, a_{d}\right) \in F^{d}$.

(3) If $\mathfrak{p}$ is prime, then so is $\mathfrak{p}+\boldsymbol{c}$.

Let $\mathfrak{p}$ be a prime ideal of $F[s]$. We shall introduce an equivalence relation in

$$
[\mathfrak{p}]:=\left\{\mathfrak{p}+\boldsymbol{c}: \boldsymbol{c} \in \mathbb{Z}^{d}\right\} .
$$

Notation. We write $\mathfrak{p} \preceq_{[\mathfrak{p}]} \mathfrak{p}+\boldsymbol{c}$ (or simply $\mathfrak{p} \preceq \mathfrak{p}+\boldsymbol{c}$ ) if $\mathbb{I}(\Omega(\boldsymbol{c})) \not \mathfrak{p}$.

Recall that a binary relation is called a preorder if it is reflexive and transitive.

Proposition 3.2. The relation $\preceq_{[\mathfrak{p}]}$ is a preorder in the set $[\mathfrak{p}]$.

Proof. First, since $\mathbb{I}(\Omega(\mathbf{0}))=(1) \nsubseteq \mathfrak{p}$, we have $\mathfrak{p} \preceq_{[\mathfrak{p}]} \mathfrak{p}$.

Second, let $\mathfrak{p} \preceq_{[\mathfrak{p}]} \mathfrak{p}+\boldsymbol{a}$ and $\mathfrak{p}+\boldsymbol{a} \preceq_{[\mathfrak{p}]} \mathfrak{p}+\boldsymbol{a}+\boldsymbol{b}$. Then we have $\mathbb{I}(\Omega(\boldsymbol{a})) \nsubseteq \mathfrak{p}$ and $\mathbb{I}(\Omega(\boldsymbol{b})) \nsubseteq \mathfrak{p}+\boldsymbol{a}$. The latter is equivalent to $\mathbb{I}(\Omega(\boldsymbol{b})-\boldsymbol{a}) \nsubseteq \mathfrak{p}$. Since $\mathfrak{p}$ is prime, we have

$$
\mathbb{I}(\Omega(\boldsymbol{a})) \mathbb{I}(\Omega(\boldsymbol{b})-\boldsymbol{a}) \nsubseteq \mathfrak{p} .
$$

From the inclusion $D\left(R_{A}\right)_{\boldsymbol{b}} D\left(R_{A}\right)_{\boldsymbol{a}} \subseteq D\left(R_{A}\right)_{\boldsymbol{a}+\boldsymbol{b}}$, we obtain

$$
\mathbb{I}(\Omega(\boldsymbol{a})) \mathbb{I}(\Omega(\boldsymbol{b})-\boldsymbol{a}) \subseteq \mathbb{I}(\Omega(\boldsymbol{a}+\boldsymbol{b})) .
$$

Hence we have

$$
\mathbb{I}(\Omega(\boldsymbol{a}+\boldsymbol{b})) \nsubseteq \mathfrak{p}
$$

or equivalently

$$
\mathfrak{p} \preceq_{[\mathfrak{p}]} \mathfrak{p}+\boldsymbol{a}+\boldsymbol{b}
$$

Let $\boldsymbol{a}, \boldsymbol{b} \in F^{d}$. In (Saito, Traves, 2001, Definition 4.1.1) a preorder $\boldsymbol{a} \preceq \boldsymbol{b}$ was defined by $E_{\tau}(\boldsymbol{a}) \subseteq E_{\tau}(\boldsymbol{b})$ for all faces $\tau$ of the cone $\mathbb{R}_{\geq 0} A$ generated by $A$, where

$$
E_{\tau}(\boldsymbol{a})=\{\boldsymbol{\lambda} \in F(A \cap \tau) / \mathbb{Z}(A \cap \tau): \boldsymbol{a}-\boldsymbol{\lambda} \in \mathbb{N} A+\mathbb{Z}(A \cap \tau)\} .
$$

Here $\mathbb{Z}(A \cap \tau)$ and $F(A \cap \tau)$ are the abelian group and the vector space generated by $A \cap \tau$, respectively. Our preorder is its generalization in the following sense.

Proposition 3.3. $\mathfrak{m}_{\boldsymbol{a}} \preceq \mathfrak{m}_{\boldsymbol{b}}$ if and only if $\boldsymbol{a} \preceq \boldsymbol{b}$. 
Proof.

$$
\begin{aligned}
\mathfrak{m}_{\boldsymbol{a}} \preceq \mathfrak{m}_{b} & \Leftrightarrow \mathfrak{m}_{\boldsymbol{a}} \preceq \mathfrak{m}_{\boldsymbol{a}}+(\boldsymbol{b}-\boldsymbol{a}) \\
& \Leftrightarrow \mathbb{I}(\Omega(\boldsymbol{b}-\boldsymbol{a})) \nsubseteq \mathfrak{m}_{\boldsymbol{a}} \\
& \Leftrightarrow \boldsymbol{a} \notin \mathrm{ZC}(\Omega(\boldsymbol{b}-\boldsymbol{a})) \\
& \Leftrightarrow \boldsymbol{a} \preceq \boldsymbol{b} .
\end{aligned}
$$

Here ZC stands for taking Zariski closure in $F^{d}$, and the last equivalence follows from (Saito, Traves, 2001, Lemma 4.1.4).

Notation. The preorder $\preceq_{[\mathfrak{p}]}$ induces an equivalence relation in $[\mathfrak{p}]$; we write $\mathfrak{p} \sim_{[\mathfrak{p}]} \mathfrak{p}+\boldsymbol{c}$ (or simply $\mathfrak{p} \sim \mathfrak{p}+\boldsymbol{c}$ ) if $\mathfrak{p} \preceq_{[\mathfrak{p}]} \mathfrak{p}+\boldsymbol{c}$ and $\mathfrak{p}+\boldsymbol{c} \preceq_{[\mathfrak{p}]} \mathfrak{p}$.

Remark 3.4. In (Musson, Van den Bergh, 1998, Lemma 3.1.9 (3)), a similar preorder $\boldsymbol{a} \underset{\boldsymbol{c}}{\Rightarrow} \boldsymbol{b}$ was defined by $\mathbb{I}(\Omega(\boldsymbol{b}-\boldsymbol{a})-(\boldsymbol{a}-\boldsymbol{c})) \mathbb{I}(\Omega(\boldsymbol{a}-\boldsymbol{c})) \nsubseteq \mathbb{I}(\Omega(\boldsymbol{b}-\boldsymbol{c})) \mathfrak{m}_{\boldsymbol{c}}$. The preorders $\boldsymbol{a} \stackrel{\boldsymbol{c}}{\preceq} \boldsymbol{b}$ and $\boldsymbol{a} \underset{\boldsymbol{c}}{\Rightarrow} \boldsymbol{b}$ induce the same equivalence relation (Musson, Van den Bergh, 1998, Lemma 3.1.9 (5); Saito, 2007, Theorem 4.10).

Proposition 3.5. Let $\mathfrak{p} \subseteq \mathfrak{q}$ be prime ideals. Then, for $\boldsymbol{c} \in \mathbb{Z}^{d}$, $\mathfrak{q} \preceq_{[\mathfrak{q}]} \mathfrak{q}+\boldsymbol{c}$ implies $\mathfrak{p} \preceq_{[\mathfrak{p}]} \mathfrak{p}+\boldsymbol{c}$. Hence $\mathfrak{q} \sim_{[\mathfrak{q}]} \mathfrak{q}+\boldsymbol{c}$ implies $\mathfrak{p} \sim_{[\mathfrak{p}]} \mathfrak{p}+\boldsymbol{c}$.

Proof. This follows from the fact that $\mathbb{I}(\Omega(\boldsymbol{c})) \nsubseteq \mathfrak{q}$ implies $\mathbb{I}(\Omega(\boldsymbol{c})) \nsubseteq \mathfrak{p}$.

Corollary 3.6. Let $\mathfrak{p}$ be a prime ideal of $F[s]$. Then $[\mathfrak{p}]$ has only a finite number of equivalence classes.

Proof. Let $\mathfrak{m}_{\boldsymbol{a}}$ be a maximal ideal containing $\mathfrak{p}$. Let $\mathfrak{m}_{\boldsymbol{b}} \in\left[\mathfrak{m}_{\boldsymbol{a}}\right]$, or equivalently let $\boldsymbol{b} \in \boldsymbol{a}+\mathbb{Z}^{d}$. Then, for a face $\tau$ and an element $\boldsymbol{\lambda} \in F(A \cap \tau)$,

$$
\boldsymbol{a}-\boldsymbol{\lambda} \in \mathbb{Z}^{d} \Leftrightarrow \boldsymbol{b}-\boldsymbol{\lambda} \in \mathbb{Z}^{d} .
$$

If such $\boldsymbol{\lambda}$ does not exist, then $E_{\tau}(\boldsymbol{a})=E_{\tau}(\boldsymbol{b})=\emptyset$ (see (3.1) for the definition of $E_{\tau}(\boldsymbol{a})$ ). If such $\boldsymbol{\lambda}$ exists, then $E_{\tau}(\boldsymbol{a})$ and $E_{\tau}(\boldsymbol{b})$ are contained in the finite set $(\boldsymbol{\lambda}+\mathbb{Q}(A \cap \tau) \cap$ $\left.\mathbb{Z}^{d}\right) / \mathbb{Z}(A \cap \tau)$ (see the proof of (Saito, 2001, Proposition $\left.2.3(1)\right)$ ). Therefore $\left[\mathfrak{m}_{\boldsymbol{a}}\right]$ has only a finite number of equivalence classes by Proposition 3.3. Hence by Proposition 3.5 we conculde that $[\mathfrak{p}]$ also has only a finite number of equivalence classes.

\section{Categories $\widetilde{\mathcal{O}}$}

We say that a left (resp. right) $D\left(R_{A}\right)$-module $M$ is $\mathbb{Z}^{d}$-graded if $M$ has a decomposition $M=\bigoplus_{\boldsymbol{b} \in \mathbb{Z}^{d}} M_{\boldsymbol{b}}$ satisfying $D\left(R_{A}\right)_{\boldsymbol{a}} M_{\boldsymbol{b}} \subseteq M_{\boldsymbol{a}+\boldsymbol{b}}\left(\operatorname{resp} . M_{\boldsymbol{b}} D\left(R_{A}\right)_{\boldsymbol{a}} \subseteq M_{\boldsymbol{a}+\boldsymbol{b}}\right)$ for all $\boldsymbol{a} \in \mathbb{Z}^{d}$ and all $\boldsymbol{b} \in \mathbb{Z}^{d}$.

Notation. Let $\widetilde{\mathcal{O}}$ (resp. ${ }^{R} \widetilde{\mathcal{O}}$ ) denote the category of $\mathbb{Z}^{d}$-graded finitely generated left (resp. right) $D\left(R_{A}\right)$-modules $M=\bigoplus_{\boldsymbol{a} \in \mathbb{Z}^{d}} M_{\boldsymbol{a}}$.

Lemma 4.1. Let $M=\bigoplus_{\boldsymbol{a} \in \mathbb{Z}^{d}} M_{\boldsymbol{a}} \in \widetilde{\mathcal{O}}$ (resp. $M \in{ }^{R} \widetilde{\mathcal{O}}$ ). Then each $M_{\boldsymbol{a}}$ is a finitely generated $F[s]$-module.

Proof. Suppose that $M$ is generated by $v_{1}, \ldots, v_{m}$ with $v_{j} \in M_{\boldsymbol{b}_{j}}(j=1, \ldots, m)$. Then the assertion is clear from the fact that each $D\left(R_{A}\right)_{\boldsymbol{a}-\boldsymbol{b}_{j}}$ is finitely generated as a $F[s]$ module. 
Notation. For $M=\bigoplus_{\boldsymbol{a} \in \mathbb{Z}^{d}} M_{\boldsymbol{a}} \in \widetilde{\mathcal{O}}\left(\operatorname{resp} .{ }^{R} \widetilde{\mathcal{O}}\right)$, set

$$
\delta(M):=\max _{\boldsymbol{a}} \operatorname{dim}_{F[s]} M_{\boldsymbol{a}} .
$$

If $0 \rightarrow L \rightarrow M \rightarrow N \rightarrow 0$ is an exact sequence of $\mathbb{Z}^{d}$-graded $D\left(R_{A}\right)$-modules, then $0 \rightarrow L_{\boldsymbol{a}} \rightarrow M_{\boldsymbol{a}} \rightarrow N_{\boldsymbol{a}} \rightarrow 0$ is exact for each $\boldsymbol{a}$. Hence we have the following.

Lemma 4.2. Let $0 \rightarrow L \rightarrow M \rightarrow N \rightarrow 0$ be an exact sequence in $\widetilde{\mathcal{O}}$ (resp. ${ }^{R} \widetilde{\mathcal{O}}$ ). Then

$$
\delta(M)=\max \{\delta(L), \delta(N)\} .
$$

Lemma 4.3. Let $M=\bigoplus_{\boldsymbol{a} \in \mathbb{Z}^{d}} M_{\boldsymbol{a}} \in \widetilde{\mathcal{O}}$ (resp. $\left.{ }^{R} \widetilde{\mathcal{O}}\right)$. Suppose that $M$ is generated by $M_{\boldsymbol{b}_{1}}, \ldots, M_{\boldsymbol{b}_{m}}$. Then

$$
\delta(M)=\max _{1 \leq i \leq m} \operatorname{dim}_{F[s]} M_{\boldsymbol{b}_{i}}
$$

Proof. Let $M \in \widetilde{\mathcal{O}}$. It suffices to prove that $\operatorname{dim} M_{\boldsymbol{b}} \geq \operatorname{dim} D\left(R_{A}\right)_{\boldsymbol{a}} M_{\boldsymbol{b}}$ for all $\boldsymbol{a}, \boldsymbol{b} \in \mathbb{Z}^{d}$. It follows from $D\left(R_{A}\right)_{\boldsymbol{a}}=t^{\boldsymbol{a}} \mathbb{I}(\Omega(\boldsymbol{a})) \subseteq t^{\boldsymbol{a}} F[s]$ that

$$
\left(\operatorname{Ann}_{F[s]}\left(M_{\boldsymbol{b}}\right)+\boldsymbol{a}\right) \subseteq \operatorname{Ann}_{F[s]}\left(D\left(R_{A}\right)_{\boldsymbol{a}} M_{\boldsymbol{b}}\right) .
$$

(resp. $\left(\operatorname{Ann}_{F[s]}\left(M_{\boldsymbol{b}}\right)-\boldsymbol{a}\right) \subseteq \operatorname{Ann}_{F[s]}\left(M_{\boldsymbol{b}} D\left(R_{A}\right)_{\boldsymbol{a}}\right)$ for a right module.)

(Here $\operatorname{Ann}_{F[s]}\left(M_{\boldsymbol{b}}\right)$ denotes the annihilator of the $F[s]$-module $M_{\boldsymbol{b}}$.) Hence $\mathfrak{p}+\boldsymbol{a} \in$ $\operatorname{Supp}\left(D\left(R_{A}\right)_{\boldsymbol{a}} M_{\boldsymbol{b}}\right)\left(\right.$ resp. $\mathfrak{p}-\boldsymbol{a} \in \operatorname{Supp}\left(M_{\boldsymbol{b}} D\left(R_{A}\right)_{\boldsymbol{a}}\right)$ for a right module) implies $\mathfrak{p} \in$ $\operatorname{Supp}\left(M_{\boldsymbol{b}}\right)$. We have thus proved the assertion.

Notation. Let $\mathfrak{p}$ be a prime ideal of $F[s]$. Let $\widetilde{\mathcal{O}}_{\mathfrak{p}}$ (resp. ${ }^{R} \widetilde{\mathcal{O}}_{\mathfrak{p}}$ ) denote the subcategory of $\widetilde{\mathcal{O}}$ (resp. ${ }^{R} \widetilde{\mathcal{O}}$ ) consisting of $M=\bigoplus_{\boldsymbol{a} \in \mathbb{Z}^{d}} M_{\boldsymbol{a}}$ such that, for all $\boldsymbol{a} \in \mathbb{Z}^{d}$, all minimal primes of the finitely generated $F[s]$-module $M_{\boldsymbol{a}}$ contain $\mathfrak{p}+\boldsymbol{a}($ resp. $\mathfrak{p}-\boldsymbol{a})$.

Remark 4.4. Let $\boldsymbol{a} \in \mathbb{Z}^{d}$. Then by definition $M \in \widetilde{\mathcal{O}}_{\mathfrak{p}}$ if and only if $M[\boldsymbol{a}] \in \widetilde{\mathcal{O}}_{\mathfrak{p}+\boldsymbol{a}}$ (resp. $M \in{ }^{R} \widetilde{\mathcal{O}}_{\mathfrak{p}}$ if and only if $\left.M[\boldsymbol{a}] \in{ }^{R} \widetilde{\mathcal{O}}_{\mathfrak{p}-\boldsymbol{a}}\right)$, where $M[\boldsymbol{a}]$ is defined by $M[\boldsymbol{a}]_{\boldsymbol{b}}=M_{\boldsymbol{a}+\boldsymbol{b}}$ for all $\boldsymbol{b} \in \mathbb{Z}^{d}$.

Remark 4.5. Let $\boldsymbol{\beta} \in F^{d}$. Then the category $\widetilde{\mathcal{O}}_{\mathfrak{m}_{\beta}}$ consists of a finitely generated $\mathbb{Z}^{d}$ graded $D\left(R_{A}\right)$-module $M=\bigoplus_{\boldsymbol{a} \in \mathbb{Z}^{d}} M_{\boldsymbol{a}}$ such that, for all $\boldsymbol{a},\left(\mathfrak{m}_{\boldsymbol{\beta}+\boldsymbol{a}}\right)^{n} M_{\boldsymbol{a}}=0(n \gg 0)$. An object of $\widetilde{\mathcal{O}}_{\mathfrak{m}_{\boldsymbol{\beta}}}$ is an object of the category $\mathcal{O}_{\boldsymbol{\beta}+\mathbb{Z}^{d}}^{(\infty)}$ in (Musson, Van den Bergh, 1998, p. 12).

Notation. Let $M \in \widetilde{\mathcal{O}}\left(\operatorname{resp} .{ }^{R} \widetilde{\mathcal{O}}\right)$, and $\delta \in \mathbb{N}$. Set

$\operatorname{Supp}_{\delta}(M):=\left\{\mathfrak{p} \in \operatorname{Spec} F[s]: \operatorname{dim} F[s] / \mathfrak{p}=\delta, \mathfrak{p}+\boldsymbol{a} \in \operatorname{Supp}\left(M_{\boldsymbol{a}}\right)\right.$ for some $\left.\boldsymbol{a}\right\}$.

$\left(\operatorname{resp} .{ }^{R} \operatorname{Supp}_{\delta}(M):=\left\{\mathfrak{p} \in \operatorname{Spec} F[s]: \operatorname{dim} F[s] / \mathfrak{p}=\delta, \mathfrak{p}-\boldsymbol{a} \in \operatorname{Supp}\left(M_{\boldsymbol{a}}\right)\right.\right.$ for some $\left.\boldsymbol{a}\right\}$.)

Proposition 4.6. Let $M=\bigoplus_{\boldsymbol{a} \in \mathbb{Z}^{d}} M_{\boldsymbol{a}} \in \widetilde{\mathcal{O}}\left(\right.$ resp. $\left.{ }^{R} \widetilde{\mathcal{O}}\right)$. Then the set $\operatorname{Supp}_{\delta(M)}(M)$ (resp. $\left.{ }^{R} \operatorname{Supp}_{\delta(M)}(M)\right)$ is finite. 
Proof. Let $M \in \widetilde{\mathcal{O}}$. Suppose that $M$ is generated by $M_{\boldsymbol{b}_{1}}, \ldots, M_{\boldsymbol{b}_{j}}$. Let $\left\{\mathfrak{p}_{k l}: l\right\}$ be the set of minimal primes of the $F[s]$-module $M_{\boldsymbol{b}_{k}}$. Suppose that $\mathfrak{q} \in \operatorname{Supp}_{F[s]}\left(M_{\boldsymbol{a}}\right)$. Then there exists $k$ such that $\mathfrak{q} \in \operatorname{Supp}_{F[s]}\left(D\left(R_{A}\right)_{\boldsymbol{a}-\boldsymbol{b}_{k}} M_{\boldsymbol{b}_{k}}\right)$. By the inclusion (4.1), we have $\mathfrak{q}+\boldsymbol{b}_{k}-\boldsymbol{a} \in \operatorname{Supp}\left(M_{\boldsymbol{b}_{k}}\right)$. Hence there exists $l$ such that $\mathfrak{q}+\boldsymbol{b}_{k}-\boldsymbol{a} \supseteq \mathfrak{p}_{k l}$, or $\mathfrak{q} \supseteq\left(\mathfrak{p}_{k l}-\boldsymbol{b}_{k}\right)+\boldsymbol{a}$. Hence we have

$$
\operatorname{Supp}_{\delta(M)}(M)=\left\{\mathfrak{p}_{k l}-\boldsymbol{b}_{k}: \operatorname{dim} F[s] / \mathfrak{p}_{k l}=\delta(M)\right\} .
$$

(For $M \in{ }^{R} \widetilde{\mathcal{O}},{ }^{R} \operatorname{Supp}_{\delta(M)}(M)=\left\{\mathfrak{p}_{k l}+\boldsymbol{b}_{k}: \operatorname{dim} F[s] / \mathfrak{p}_{k l}=\delta(M)\right\}$, respectively.)

\section{Degree of modules}

Let $\tau: R \rightarrow R^{\prime}$ be an isomorphism of commutative rings. Let $M$ be an $R$-module, $N$ an $R^{\prime}$-module. A $\mathbb{Z}$-module homomorphism $f: M \rightarrow N$ is called a $\tau$-homomorphism if $f(a m)=\tau(a) f(m)$ for $a \in R$ and $m \in M$.

Let $\tau: R \rightarrow R$ be an automorphism, and $f: M \rightarrow N$ a $\tau$-homomorphism. Let $\mathfrak{p}$ be a prime ideal of $R$. Then $\tau(\mathfrak{p})$ is also a prime ideal of $R$, and

$$
\tau_{\mathfrak{p}}: R_{\mathfrak{p}} \ni \frac{a}{s} \mapsto \frac{\tau(a)}{\tau(s)} \in R_{\tau(\mathfrak{p})}
$$

is an isomorphism of rings. Furthermore $f$ induces $\tau_{\mathfrak{p}}$-homomorphism

$$
f_{\mathfrak{p}}: M_{\mathfrak{p}} \ni \frac{m}{s} \mapsto \frac{f(m)}{\tau(s)} \in N_{\tau(\mathfrak{p})} .
$$

Clearly, if $L$ is an $R$-submodule of $M$, then $f_{\mathfrak{p}}\left(L_{\mathfrak{p}}\right)$ is an $R_{\tau(\mathfrak{p}) \text {-submodule of }} N_{\tau(\mathfrak{p})}$.

Example 5.1. Let $\boldsymbol{a} \in \mathbb{Z}^{d}$, and let $\tau_{\boldsymbol{a}}$ be an automorphism of $F[s]$ defined by $f(s) \mapsto$ $f(s+\boldsymbol{a})$. Let $Q=t^{\boldsymbol{a}} q(s) \in D\left(R_{A}\right)_{\boldsymbol{a}}$, and let $M$ be a $\mathbb{Z}^{d}$-graded left $D\left(R_{A}\right)$-module. Then $Q \times: M_{\boldsymbol{b}} \ni m \mapsto Q m \in M_{\boldsymbol{a}+\boldsymbol{b}}$ is a $\tau_{-\boldsymbol{a}}$-homomorphism; for $g(s) \in F[s]$ and $m \in M_{\boldsymbol{b}}$

$$
Q(g(s) m)=t^{\boldsymbol{a}} q(s) g(s) m=g(s-\boldsymbol{a}) t^{\boldsymbol{a}} q(s) m=g(s-\boldsymbol{a}) Q(m) .
$$

For a $\mathbb{Z}^{d}$-graded right $D\left(R_{A}\right)$-module $M, \times Q: M_{\boldsymbol{b}} \ni m \mapsto m Q \in M_{\boldsymbol{a}+\boldsymbol{b}}$ is a $\tau_{\boldsymbol{a}^{-}}$ homomorphism.

Let $R$ be a commutative Noetherian ring, and $N$ a finitely generated $R$-module. If $\mathfrak{p}$ is a minimal prime of $N$, then $N_{\mathfrak{p}}$ is an Artinian $R_{\mathfrak{p}}$-module, and $N_{\mathfrak{p}}$ has a composition series (see e.g. Eisenbud, 1995, Corollary 2.18); we denote its length by length $R_{\mathfrak{p}}\left(N_{\mathfrak{p}}\right)$.

Lemma 5.2. Let $M$ be a $\mathbb{Z}^{d}$-graded left (resp. right) $D\left(R_{A}\right)$-module. Let $\mathfrak{p}$ be a prime ideal of $F[s]$ with $\operatorname{dim} F[s] / \mathfrak{p}=\delta(M)$. Suppose that $\mathfrak{p}+\boldsymbol{a} \sim \mathfrak{p}+\boldsymbol{b}($ resp. $\mathfrak{p}-\boldsymbol{a} \sim \mathfrak{p}-\boldsymbol{b})$. Then

$$
\begin{gathered}
\operatorname{length}_{F[s]_{\mathfrak{p}+\boldsymbol{a}}}\left(M_{\boldsymbol{a}}\right)_{\mathfrak{p}+\boldsymbol{a}}=\operatorname{length}_{F[s]_{\mathfrak{p}+\boldsymbol{b}}}\left(M_{\boldsymbol{b}}\right)_{\mathfrak{p}+\boldsymbol{b}}, \\
(\text { resp. length } \\
F[s]_{\mathfrak{p}-\boldsymbol{a}} \\
\left.\left(M_{\boldsymbol{a}}\right)_{\mathfrak{p}-\boldsymbol{a}}=\operatorname{length}_{F[s]_{\mathfrak{p}-\boldsymbol{b}}}\left(M_{\boldsymbol{b}}\right)_{\mathfrak{p}-\boldsymbol{b}}\right) .
\end{gathered}
$$

Proof. From now on, we prove assertions only in the left module case. The proofs in the right module case are similar.

Since $\mathfrak{p}+\boldsymbol{a} \sim \mathfrak{p}+\boldsymbol{b}$, we have

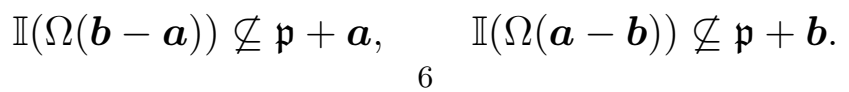


Take

$$
q_{\boldsymbol{a}}(s) \in \mathbb{I}(\Omega(\boldsymbol{b}-\boldsymbol{a})) \backslash(\mathfrak{p}+\boldsymbol{a}), \quad q_{\boldsymbol{b}}(s) \in \mathbb{I}(\Omega(\boldsymbol{a}-\boldsymbol{b})) \backslash(\mathfrak{p}+\boldsymbol{b}),
$$

and put

$$
Q_{\boldsymbol{a}}:=t^{\boldsymbol{b}-\boldsymbol{a}} q_{\boldsymbol{a}}(s), \quad Q_{\boldsymbol{b}}:=t^{\boldsymbol{a}-\boldsymbol{b}} q_{\boldsymbol{b}}(s)
$$

Then

$$
Q_{a}: M_{a} \rightarrow M_{b}, \quad Q_{b}: M_{b} \rightarrow M_{a}
$$

are a $\tau_{\boldsymbol{a}-\boldsymbol{b}}$-homomorphism and a $\tau_{\boldsymbol{b}-\boldsymbol{a}}$-homomorphism, respectively. They induce

$$
\left(Q_{\boldsymbol{a}}\right)_{\mathfrak{p}+\boldsymbol{a}}:\left(M_{\boldsymbol{a}}\right)_{\mathfrak{p}+\boldsymbol{a}} \rightarrow\left(M_{\boldsymbol{b}}\right)_{\mathfrak{p}+\boldsymbol{b}}, \quad\left(Q_{\boldsymbol{b}}\right)_{\mathfrak{p}+\boldsymbol{b}}:\left(M_{\boldsymbol{b}}\right)_{\mathfrak{p}+\boldsymbol{b}} \rightarrow\left(M_{\boldsymbol{a}}\right)_{\mathfrak{p}+\boldsymbol{a}}
$$

Furthermore we have

$$
Q_{\boldsymbol{b}} Q_{\boldsymbol{a}}=t^{\boldsymbol{a}-\boldsymbol{b}} q_{\boldsymbol{b}}(s) t^{\boldsymbol{b}-\boldsymbol{a}} q_{\boldsymbol{a}}(s)=q_{\boldsymbol{b}}(s+\boldsymbol{b}-\boldsymbol{a}) q_{\boldsymbol{a}}(s) .
$$

Since $q_{\boldsymbol{b}}(s) \notin \mathfrak{p}+\boldsymbol{b}$ means $q_{\boldsymbol{b}}(s+\boldsymbol{b}-\boldsymbol{a}) \notin \mathfrak{p}+\boldsymbol{a}$, we have

$$
Q_{\boldsymbol{b}} Q_{\boldsymbol{a}}=q_{\boldsymbol{b}}(s+\boldsymbol{b}-\boldsymbol{a}) q_{\boldsymbol{a}}(s) \notin \mathfrak{p}+\boldsymbol{a} .
$$

Similarly

$$
Q_{\boldsymbol{a}} Q_{\boldsymbol{b}}=q_{\boldsymbol{a}}(s+\boldsymbol{a}-\boldsymbol{b}) q_{\boldsymbol{b}}(s) \notin \mathfrak{p}+\boldsymbol{b} .
$$

Hence from (5.2) we obtain

$$
\operatorname{length}_{F[s]_{\mathfrak{p}+\boldsymbol{a}}}\left(M_{\boldsymbol{a}}\right)_{\mathfrak{p}+\boldsymbol{a}}=\text { length }_{F[s]_{\mathfrak{p}+\boldsymbol{b}}}\left(M_{\boldsymbol{b}}\right)_{\mathfrak{p}+\boldsymbol{b}} .
$$

Let $M \in \widetilde{\mathcal{O}}$. We know that the set $\operatorname{Supp}_{\delta(M)}(M)$ is finite by Proposition 4.6. Let $\mathfrak{p} \in \operatorname{Supp}_{\delta(M)}(M)$. Then by Lemma 5.2 if $\mathfrak{p}+\boldsymbol{a} \sim_{[\mathfrak{p}]} \mathfrak{p}+\boldsymbol{b}$, then $\operatorname{length}_{F[s]_{\mathfrak{p}+\boldsymbol{a}}} M_{\boldsymbol{a}}=$ length $_{F[s]_{\mathfrak{p}+\boldsymbol{b}}} M_{\boldsymbol{b}}$.

Notation. Let $M \in \widetilde{\mathcal{O}}$ (resp. $\left.{ }^{R} \widetilde{\mathcal{O}}\right)$. Set

$$
\begin{gathered}
\operatorname{deg} M:=\sum_{\mathfrak{p} \in \operatorname{Supp}_{\delta(M)}(M)} \sum_{\mathfrak{p}+\boldsymbol{a} \in[\mathfrak{p}] / \sim_{[\mathfrak{p}]}} \operatorname{length}_{F[s]_{\mathfrak{p}+\boldsymbol{a}}} M_{\boldsymbol{a}} . \\
\text { (resp. } \left.\operatorname{deg} M:=\sum_{\mathfrak{p} \in \operatorname{Supp}_{\delta(M)}(M)} \sum_{\mathfrak{p}-\boldsymbol{a} \in[\mathfrak{p}] / \sim_{[\mathfrak{p}]}} \operatorname{length}_{F[s]_{\mathfrak{p}-\boldsymbol{a}}} M_{\boldsymbol{a}} .\right)
\end{gathered}
$$

The following lemma is clear from the definitions.

Lemma 5.3. Let $M \in \widetilde{\mathcal{O}}\left(r e s p .{ }^{R} \widetilde{\mathcal{O}}\right)$.

(1) If $M \neq 0$, then $\operatorname{deg} M \neq 0$.

(2) Let $0 \rightarrow L \rightarrow M \rightarrow N \rightarrow 0$ be an exact sequence in $\widetilde{\mathcal{O}}$ (resp. $\left.{ }^{R} \widetilde{\mathcal{O}}\right)$. Then

(a) If $\delta(M)=\delta(N)$, then $\operatorname{deg} M \geq \operatorname{deg} N$.

(b) If $\delta(M)=\delta(N)$ and $\operatorname{deg} M=\operatorname{deg} N$, then $\delta(L)<\delta(M)$.

(c) If $\delta(L)=\delta(M)$, then $\operatorname{deg} L \leq \operatorname{deg} M$.

(d) If $\delta(L)=\delta(M)$ and $\operatorname{deg} L=\operatorname{deg} M$, then $\delta(N)<\delta(M)$.

(3) Let

$$
M=M_{0} \supseteq M_{1} \supseteq M_{2} \supseteq \cdots \supseteq M_{i} \supseteq \cdots
$$

be a descending chain in $\widetilde{\mathcal{O}}$ (resp. $\left.{ }^{R} \widetilde{\mathcal{O}}\right)$. Then there exist at most $\operatorname{deg} M i$ 's such that $\delta\left(M_{i} / M_{i+1}\right)=\delta(M)$. 


\section{KRull Dimension}

In this section we consider the Krull dimension for the category $\widetilde{\mathcal{O}}$ (resp. ${ }^{R} \widetilde{\mathcal{O}}$ ) in the sense of (Gabriel, 1962) and (Rentschler, Gabriel, 1967), which we simply call it Krull dimension.

Notation. Let $M \in \widetilde{\mathcal{O}}$ (resp. ${ }^{R} \widetilde{\mathcal{O}}$ ). Then we denote by $\operatorname{Kdim} M$ the Krull dimension for the lattice of $\mathbb{Z}^{d}$-graded $D\left(R_{A}\right)$-submodules.

Theorem 6.1. Let $M \in \widetilde{\mathcal{O}}\left(r e s p .{ }^{R} \widetilde{\mathcal{O}}\right)$. Then

$$
\operatorname{Kdim} M=\delta(M) \text {. }
$$

Proof. Let $M \in \widetilde{\mathcal{O}}$.

First $\delta(M)<0$ if and only if $M=0$ if and only if $\operatorname{Kdim} M<0$.

Next let $M \neq 0$, and let $\delta:=\delta(M)$. We prove $\operatorname{Kdim} M=\delta$ by induction on $\delta$.

By Lemma 4.2 and (McConnel-Robson, 1987, Lemma 6.2.4) we may assume that $M$ is cyclic. Furthermore, by shifting its grading if necessary, we may assume that $M=$ $D\left(R_{A}\right) M_{\mathbf{0}}$. We know $\delta=\operatorname{dim}_{F[s]} M_{\mathbf{0}}$ by Lemma 4.3 .

Let $\mathfrak{p}_{1}, \ldots, \mathfrak{p}_{l}$ be the minimal primes of $M_{0}$ with $\operatorname{dim} F[s] / \mathfrak{p}_{j}=\delta$ for $j \leq k$, and $<\delta$ for $j>k$. Then $\operatorname{Supp}_{\delta}(M)=\left\{\mathfrak{p}_{1}, \ldots, \mathfrak{p}_{k}\right\}$. By Lemma 4.2 and (McConnel-Robson, 1987, Lemma 6.2.4) again, we may assume that $k=1$ and $\operatorname{deg} M=1$.

Let

$$
M=M_{0} \supseteq M_{1} \supseteq \cdots \supseteq M_{m} \supseteq \cdots
$$

be a descending chain of $\mathbb{Z}^{d}$-graded $D\left(R_{A}\right)$-modules. Suppose that $\delta\left(M_{i} / M_{i+1}\right)=\delta$. Then clearly $\delta\left(M / M_{i+1}\right)=\delta$. Since $\operatorname{deg} M / M_{i+1}=1=\operatorname{deg} M$, by Lemma $5.3 \delta\left(M_{i+1}\right)<\delta$. Hence $\delta\left(M_{j} / M_{j+1}\right)<\delta$ for all $j>i$. By the induction hypothesis, $\operatorname{Kdim}\left(M_{j} / M_{j+1}\right)<\delta$ for all $j>i$. Therefore we have proved that $\operatorname{Kdim} M \leq \delta$.

Finally we prove the inequality $\operatorname{Kdim} M \geq \delta$. Let $N_{1} \subsetneq N_{2}$ be proper $F[s]$-submodules of $M_{\mathbf{0}}$. Then $D\left(R_{A}\right) N_{1} \subsetneq D\left(R_{A}\right) N_{2}$ are proper $\mathbb{Z}^{d}$-graded $D\left(R_{A}\right)$-submodules of $M$, since $\left(D\left(R_{A}\right) N_{i}\right)_{\mathbf{0}}=N_{i}(i=1,2)$. Hence

$$
\operatorname{Kdim} M \geq \operatorname{dim}_{F[s]} M_{0}=\delta .
$$

The following is clear from Theorem 6.1, and also from the non-graded version (Musson, Van den Bergh, 1998, Theorem 8.1.2).

Corollary 6.2. As $D\left(R_{A}\right) \in \widetilde{\mathcal{O}}\left(\operatorname{resp} .{ }^{R} \widetilde{\mathcal{O}}\right)$,

$$
\operatorname{Kdim} D\left(R_{A}\right)=d .
$$

\section{CRitical modules}

Let $R$ be a left (resp. right) Noetherian ring. An $R$-module $M$ of Krull dimension $\delta$ is said to be $\delta$-critical if $\operatorname{Kdim}(M / N)<\delta$ for all nonzero $R$-submodules $N$ of $M$. The 0 -critical modules are precisely the simple modules. Hence critical modules play a fundamental role in the theory of Noetherian modules. (See for example (Goodearl, Warfield, 1989; Lenagan, 2000; McConnel, Robson 1987).) In this section, we characterize critical $\mathbb{Z}^{d}$-graded $D\left(R_{A}\right)$-modules (Theorem 7.4 ), and explicitly describe cyclic ones (Theorem 7.7).

The following lemma is clear from the definitions. 
Lemma 7.1. Let $R$ be a commutative Noetherian ring, and $M$ a finitely generated $R$ module. Then $M$ is critical if and only if there exists a prime ideal $\mathfrak{p}$ of $R$ such that $\operatorname{Ass}(M)=\{\mathfrak{p}\}$, and length $R_{\mathfrak{p}}\left(M_{\mathfrak{p}}\right)=1$, where $\operatorname{Ass}(M)$ denotes the set of associated primes of $M$. If this is the case, then $\operatorname{Ann}_{R} M=\mathfrak{p}$.

Lemma 7.2. Suppose that $M$ is a $\delta$-critical $\mathbb{Z}^{d}$-graded left (resp. right) $D\left(R_{A}\right)$-module. Then for each $\boldsymbol{a} \in \mathbb{Z}^{d}$ the $F[s]$-module $M_{\boldsymbol{a}}$ is $\delta$-critical if it is not zero.

Proof. First suppose that $\operatorname{dim} M_{\boldsymbol{a}}=\delta$. Let $N$ be a nonzero $F[s]$-submodule of $M_{\boldsymbol{a}}$ with $\operatorname{dim} M_{\boldsymbol{a}} / N=\delta^{\prime}$. Then by Theorem 6.1

$$
\operatorname{Kdim}\left(M / D\left(R_{A}\right) N\right) \geq \operatorname{dim}_{F[s]}\left(M / D\left(R_{A}\right) N\right)_{\boldsymbol{a}}=\operatorname{dim}_{F[s]}\left(M_{\boldsymbol{a}} / N\right)=\delta^{\prime} .
$$

Since $M$ is $\delta$-critical, $\delta^{\prime}<\delta$. Hence $M_{\boldsymbol{a}}$ is $\delta$-critical.

Next suppose that $0 \neq \operatorname{dim}_{F[s]} M_{\boldsymbol{a}}<\delta$. Then there exists $\boldsymbol{b}$ such that $\operatorname{Kdim} M=$ $\operatorname{dim}_{F[s]} M_{\boldsymbol{b}}=\delta$. Consider the submodule $D\left(R_{A}\right) M_{\boldsymbol{a}}$ of $M$. Then

$$
\operatorname{Kdim} M / D\left(R_{A}\right) M_{\boldsymbol{a}} \geq \operatorname{dim}_{F[s]}\left(M / D\left(R_{A}\right) M_{\boldsymbol{a}}\right)_{\boldsymbol{b}}=\operatorname{dim}_{F[s]}\left(M_{\boldsymbol{b}} / D\left(R_{A}\right)_{\boldsymbol{b}-\boldsymbol{a}} M_{\boldsymbol{a}}\right) .
$$

We know by the proof of Lemma 4.3

$$
\operatorname{dim}_{F[s]} D\left(R_{A}\right)_{\boldsymbol{b}-\boldsymbol{a}} M_{\boldsymbol{a}} \leq \operatorname{dim}_{F[s]} M_{\boldsymbol{a}}<\delta .
$$

Hence

$$
\operatorname{Kdim} M / D\left(R_{A}\right) M_{\boldsymbol{a}} \geq \operatorname{dim}_{F[s]} M_{\boldsymbol{b}}=\delta,
$$

which contradicts the fact that $M$ is critical. Hence there exists no nonzero $M_{\boldsymbol{a}}$ with $\operatorname{dim}_{F[s]} M_{\boldsymbol{a}}<\delta$.

Let $M$ be a critical $\mathbb{Z}^{d}$-graded left (resp. right) $D\left(R_{A}\right)$-module with $M_{\boldsymbol{a}} \neq 0$. Then by Lemmas 7.1 and 7.2 there exists a prime ideal $\mathfrak{p}$ of $F[s]$ such that $\operatorname{Ass}\left(M_{\boldsymbol{a}}\right)=\{\mathfrak{p}\}$. By considering the shift $M[\boldsymbol{a}]$, we may assume that $M_{\mathbf{0}} \neq 0$.

Proposition 7.3. Let $\mathfrak{p}$ be a prime ideal of $F[s]$ with $\operatorname{dim} F[s] / \mathfrak{p}=\delta$. Suppose that $M$ is a $\delta$-critical $\mathbb{Z}^{d}$-graded left (resp. right) $D\left(R_{A}\right)$-module with $\operatorname{Ass}\left(M_{\mathbf{0}}\right)=\{\mathfrak{p}\}$. Then

(1) $M \in \widetilde{\mathcal{O}}_{\mathfrak{p}}$ (resp. $\left.M \in{ }^{R} \widetilde{\mathcal{O}}_{\mathfrak{p}}\right)$.

(2) $M_{\boldsymbol{a}}=0$ if $\mathfrak{p} \nsim \mathfrak{p}+\boldsymbol{a}($ resp. $\mathfrak{p} \nsim \mathfrak{p}-\boldsymbol{a})$.

(3) $\operatorname{Ass}\left(M_{\boldsymbol{a}}\right)=\{\mathfrak{p}+\boldsymbol{a}\}$ if $\mathfrak{p} \sim \mathfrak{p}+\boldsymbol{a} .\left(\right.$ resp. $\operatorname{Ass}\left(M_{\boldsymbol{a}}\right)=\{\mathfrak{p}-\boldsymbol{a}\}$ if $\left.\mathfrak{p} \sim \mathfrak{p}-\boldsymbol{a}.\right)$

Proof. Suppose that $D\left(R_{A}\right)_{-\boldsymbol{a}} M_{\boldsymbol{a}}=0$. Then

$$
\operatorname{dim}_{F[s]}\left(M / D\left(R_{A}\right) M_{\boldsymbol{a}}\right)_{\mathbf{0}}=\operatorname{dim}_{F[s]}\left(M_{\mathbf{0}} / D\left(R_{A}\right)_{-\boldsymbol{a}} M_{\boldsymbol{a}}\right)=\operatorname{dim}_{F[s]}\left(M_{\mathbf{0}}\right)=\delta,
$$

which means $\operatorname{Kdim}\left(M / D\left(R_{A}\right) M_{\boldsymbol{a}}\right)=\delta$. Since $M$ is $\delta$-critical, $D\left(R_{A}\right) M_{\boldsymbol{a}}=0$, and hence $M_{a}=0$.

Let $M_{\boldsymbol{a}} \neq 0$, and $\mathfrak{q} \in \operatorname{Ass}\left(M_{\boldsymbol{a}}\right)$. Then by Lemmas 7.1 and 7.2 we have $\operatorname{Ass}\left(M_{\boldsymbol{a}}\right)=\{\mathfrak{q}\}$ and $\mathfrak{q}=\operatorname{Ann}_{F[s]} M_{\boldsymbol{a}}$. By (4.1)

$$
\operatorname{Ann}_{F[s]}\left(D\left(R_{A}\right)_{-\boldsymbol{a}} M_{\boldsymbol{a}}\right) \supseteq \operatorname{Ann}_{F[s]} M_{\boldsymbol{a}}-\boldsymbol{a}=\mathfrak{q}-\boldsymbol{a} .
$$

By the first paragraph of the proof, $D\left(R_{A}\right)_{-\boldsymbol{a}} M_{\boldsymbol{a}} \neq 0$. Since $\operatorname{Ann}_{F[s]}(x)=\mathfrak{p}$ for each nonzero element $x \in D\left(R_{A}\right)_{-\boldsymbol{a}} M_{\boldsymbol{a}} \subseteq M_{\mathbf{0}}$, we have

$$
\operatorname{Ann}_{F[s]}\left(D\left(R_{A}\right)_{-\boldsymbol{a}} M_{\boldsymbol{a}}\right)=\mathfrak{p} .
$$

Hence $\mathfrak{q} \subseteq \mathfrak{p}+\boldsymbol{a}$. Then $\mathfrak{q}=\mathfrak{p}+\boldsymbol{a}$, since $\operatorname{dim} F[s] / \mathfrak{p}=\operatorname{Kdim} M$, and thus $\operatorname{Ass}\left(M_{\boldsymbol{a}}\right)=$ $\{\mathfrak{p}+\boldsymbol{a}\}$. 
Suppose that $\mathfrak{p} \sim \mathfrak{p}+\boldsymbol{a}$. Then by Lemma $5.2 M_{\boldsymbol{a}} \neq 0$. Hence $\operatorname{Ass}\left(M_{\boldsymbol{a}}\right)=\{\mathfrak{p}+\boldsymbol{a}\}$ by the previous paragraph.

Finally suppose that $\mathfrak{p} \not \mathfrak{p}+\boldsymbol{a}$. Then $\mathbb{I}(\Omega(\boldsymbol{a})) \subseteq \mathfrak{p}$ or $\mathbb{I}(\Omega(-\boldsymbol{a})) \subseteq \mathfrak{p}+\boldsymbol{a}$. Hence $\mathbb{I}(\Omega(-\boldsymbol{a})-\boldsymbol{a}) \mathbb{I}(\Omega(\boldsymbol{a})) \subseteq \mathfrak{p}$. Consider the submodule $D\left(R_{A}\right)\left(D\left(R_{A}\right)_{\boldsymbol{a}} M_{\mathbf{0}}\right)$ of $M$. We have

$$
\begin{aligned}
\left(D\left(R_{A}\right)\left(D\left(R_{A}\right)_{\boldsymbol{a}} M_{\mathbf{0}}\right)\right)_{\mathbf{0}} & =D\left(R_{A}\right)_{-\boldsymbol{a}} D\left(R_{A}\right)_{\boldsymbol{a}} M_{\mathbf{0}} \\
& =t^{-\boldsymbol{a}} \mathbb{I}(\Omega(-\boldsymbol{a})) t^{\boldsymbol{a}} \mathbb{I}(\Omega(\boldsymbol{a})) M_{\mathbf{0}} \\
& =\mathbb{I}(\Omega(-\boldsymbol{a})-\boldsymbol{a}) \mathbb{I}(\Omega(\boldsymbol{a})) M_{\mathbf{0}} \\
& \subseteq \mathfrak{p} M_{0}=0 .
\end{aligned}
$$

Since $\operatorname{Kdim}\left(M / D\left(R_{A}\right)\left(D\left(R_{A}\right)_{\boldsymbol{a}} M_{\mathbf{0}}\right)\right) \geq \operatorname{dim}\left(M / D\left(R_{A}\right)\left(D\left(R_{A}\right)_{\boldsymbol{a}} M_{\mathbf{0}}\right)\right)_{\mathbf{0}}=\operatorname{dim} M_{\mathbf{0}}=\delta=$ $\operatorname{Kdim} M$, and since $M$ is critical, $D\left(R_{A}\right)\left(D\left(R_{A}\right)_{\boldsymbol{a}} M_{\mathbf{0}}\right)=0$. Hence $D\left(R_{A}\right)_{\boldsymbol{a}} M_{\mathbf{0}}=0$. If $M_{\boldsymbol{a}} \neq 0$, then $\operatorname{Ass}\left(M_{\boldsymbol{a}}\right)=\{\mathfrak{p}+\boldsymbol{a}\}$, and

$\operatorname{Kdim} M / D\left(R_{A}\right) M_{\mathbf{0}} \geq \operatorname{dim}\left(M / D\left(R_{A}\right) M_{\mathbf{0}}\right)_{\boldsymbol{a}}=\operatorname{dim} M_{\boldsymbol{a}} / D\left(R_{A}\right)_{\boldsymbol{a}} M_{\mathbf{0}}=\operatorname{dim} M_{\boldsymbol{a}}=\delta$.

This contradicts the fact that $M$ is critical. Therefore $M_{\boldsymbol{a}}=0$ in this case.

Theorem 7.4. Let $M$ be a finitely generated left (resp. right) $\mathbb{Z}^{d}$-graded $D\left(R_{A}\right)$-module with $M_{\mathbf{0}} \neq 0$. Then $M$ is critical if and only if there exists a prime ideal $\mathfrak{p}$ of $F[s]$ such that

(1) $M \in \widetilde{\mathcal{O}}_{\mathfrak{p}}$ (resp. $\left.M \in{ }^{R} \widetilde{\mathcal{O}}_{\mathfrak{p}}\right)$.

(2) $M_{\boldsymbol{a}}=0$ if $\mathfrak{p} \nsim \mathfrak{p}+\boldsymbol{a}($ resp. $\mathfrak{p} \nsim \mathfrak{p}-\boldsymbol{a})$.

(3) $M_{\boldsymbol{a}}$ is a critical $F[s]$-module with $\operatorname{Ass}\left(M_{\boldsymbol{a}}\right)=\{\mathfrak{p}+\boldsymbol{a}\}$ if $\mathfrak{p} \sim \mathfrak{p}+\boldsymbol{a}\left(\right.$ resp. $\operatorname{Ass}\left(M_{\boldsymbol{a}}\right)=$ $\{\mathfrak{p}-\boldsymbol{a}\}$ if $\mathfrak{p} \sim \mathfrak{p}-\boldsymbol{a})$.

Proof. Proposition 7.3 is the only-if part.

Assume the conditions (1) through (3). Suppose that $\mathfrak{p} \sim \mathfrak{p}+\boldsymbol{a}$. Let $N$ be a nonzero $F[s]$-submodule of $M_{\boldsymbol{a}}$. Consider the quotient $M / D\left(R_{A}\right) N$.

First let $\mathfrak{p} \nsim \mathfrak{p}+\boldsymbol{b}$. Then $\left(M / D\left(R_{A}\right) N\right)_{\boldsymbol{b}}=0$, since $M_{\boldsymbol{b}}=0$.

Next let $\mathfrak{p} \sim \mathfrak{p}+\boldsymbol{b}$. Then $\mathfrak{p}+\boldsymbol{a} \sim \mathfrak{p}+\boldsymbol{b}$. By Lemma 5.2

$$
\begin{aligned}
& \text { length }_{F[s]_{\mathfrak{p}+\boldsymbol{b}}}\left(\left(D\left(R_{A}\right) N\right)_{\boldsymbol{b}}\right)_{\mathfrak{p}+\boldsymbol{b}}=\operatorname{length}_{F[s]_{\mathfrak{p}+\boldsymbol{a}}}\left(\left(D\left(R_{A}\right) N\right)_{\boldsymbol{a}}\right)_{\mathfrak{p}+\boldsymbol{a}}=\operatorname{length}_{F[s]_{\mathfrak{p}+\boldsymbol{a}}} N_{\mathfrak{p}+\boldsymbol{a}} \\
& =1 \\
& =\operatorname{length}_{F[s]_{\mathfrak{p}+\boldsymbol{a}}}\left(M_{\boldsymbol{a}}\right)_{\mathfrak{p}+\boldsymbol{a}}=\operatorname{length}_{F[s]_{\mathfrak{p}+\boldsymbol{b}}}\left(M_{\boldsymbol{b}}\right)_{\mathfrak{p}+\boldsymbol{b}} .
\end{aligned}
$$

Hence

$$
\operatorname{dim}_{F[s]}\left(M / D\left(R_{A}\right) N\right)_{\boldsymbol{b}}<\operatorname{dim}_{F[s]} M_{\boldsymbol{b}}=\operatorname{Kdim} M .
$$

Therefore $\operatorname{Kdim} M / D\left(R_{A}\right) N<\operatorname{Kdim} M$.

Example 7.5. Let $\mathfrak{p}$ be a prime ideal of $F[s]$, and let $\delta=\operatorname{dim} F[s] / \mathfrak{p}$. Let ${ }^{L} L(\mathfrak{p})=$ $\bigoplus_{\boldsymbol{a} \in \mathbb{Z}^{d}}{ }^{L} L(\mathfrak{p})_{\boldsymbol{a}}\left(\right.$ resp. $\left.{ }^{R} L(\mathfrak{p})=\bigoplus_{\boldsymbol{a} \in \mathbb{Z}^{d}} R(\mathfrak{p})_{\boldsymbol{a}}\right)$ be the cyclic $\mathbb{Z}^{d}$-graded left (resp. right) $D\left(R_{A}\right)$-module ${ }^{L} L(\mathfrak{p})=D\left(R_{A}\right) /{ }^{L} I(\mathfrak{p})\left(\right.$ resp. $\left.{ }^{R} L(\mathfrak{p})=D\left(R_{A}\right) /{ }^{R} I(\mathfrak{p})\right)$ with

$$
\begin{aligned}
{ }^{L} I(\mathfrak{p})_{\boldsymbol{a}} & = \begin{cases}t^{\boldsymbol{a}}(\mathbb{I}(\Omega(\boldsymbol{a})) \cap \mathfrak{p}) & \left(\mathfrak{p} \sim_{[\mathfrak{p}]} \mathfrak{p}+\boldsymbol{a}\right) \\
D\left(R_{A}\right)_{\boldsymbol{a}} & (\text { otherwise }) .\end{cases} \\
\left(\text { resp. }{ }^{R} I(\mathfrak{p})_{\boldsymbol{a}}\right. & =\left\{\begin{array}{ll}
(\mathbb{I}(\Omega(\boldsymbol{a})+\boldsymbol{a}) \cap \mathfrak{p}) t^{\boldsymbol{a}} & \left(\mathfrak{p} \sim_{[\mathfrak{p}]} \mathfrak{p}-\boldsymbol{a}\right) \\
D\left(R_{A}\right)_{\boldsymbol{a}} & \text { (otherwise). }
\end{array}\right)
\end{aligned}
$$

Then ${ }^{L} L(\mathfrak{p})\left(\right.$ resp. $\left.{ }^{R} L(\mathfrak{p})\right)$ is $\delta$-critical by Theorem 7.4 . 
If $\mathfrak{p}=\mathfrak{m}_{\beta}$, then

$$
\begin{aligned}
& { }^{L} L\left(\mathfrak{m}_{\boldsymbol{\beta}}\right)_{\boldsymbol{a}}=\left\{\begin{array}{ll}
t^{\boldsymbol{a}} \mathbb{I}(\Omega(\boldsymbol{a})) / t^{\boldsymbol{a}}\left(\mathbb{I}(\Omega(\boldsymbol{a})) \cap \mathfrak{m}_{\boldsymbol{\beta}}\right) & (\boldsymbol{\beta} \sim \boldsymbol{\beta}+\boldsymbol{a}) \\
0 & \text { (otherwise) }
\end{array}={ }^{L} L(\boldsymbol{\beta})_{\boldsymbol{\beta}+\boldsymbol{a}} .\right. \\
& \left(\operatorname{resp} .{ }^{R} L\left(\mathfrak{m}_{\boldsymbol{\beta}}\right)_{\boldsymbol{a}}=\left\{\begin{array}{ll}
\mathbb{I}(\Omega(\boldsymbol{a})+\boldsymbol{a}) t^{\boldsymbol{a}} /\left(\mathbb{I}(\Omega(\boldsymbol{a})+\boldsymbol{a}) \cap \mathfrak{m}_{\boldsymbol{\beta}}\right) t^{\boldsymbol{a}} & (\boldsymbol{\beta} \sim \boldsymbol{\beta}-\boldsymbol{a}) \\
0 & \text { (otherwise) }
\end{array}={ }^{R} L(\boldsymbol{\beta})_{-\boldsymbol{\beta}+\boldsymbol{a}} .\right)\right.
\end{aligned}
$$

Thus ${ }^{L} L\left(\mathfrak{m}_{\boldsymbol{\beta}}\right)={ }^{L} L(\boldsymbol{\beta})[\boldsymbol{\beta}]\left(\right.$ resp. $\left.{ }^{R} L\left(\mathfrak{m}_{\boldsymbol{\beta}}\right)={ }^{R} L(\boldsymbol{\beta})[-\boldsymbol{\beta}]\right)$. (See (Saito, 2007) for ${ }^{L} L(\boldsymbol{\beta})$ and ${ }^{R} L(\boldsymbol{\beta})$.)

Example 7.6. Let $n=d=2$, and let $A=\left\{{ }^{t}(1,0),{ }^{t}(0,1)\right\}$. Then $D=D\left(R_{A}\right)$ is the second Weyl algebra $F\left\langle t_{1}, t_{2}, \partial_{1}, \partial_{2}\right\rangle$. Let $\mathfrak{p}$ be the prime ideal $\left\langle s_{1}\right\rangle$ of $F[s]$. We know

$$
\mathbb{I}(\Omega(\boldsymbol{c}))=\left\langle\prod_{c_{i}<0} \prod_{m=0}^{-c_{i}-1}\left(s_{i}-m\right)\right\rangle .
$$

Hence

$$
\mathbb{I}(\Omega(\boldsymbol{c})) \subseteq \mathfrak{p} \Leftrightarrow c_{1}<0
$$

or equivalently

$$
\mathfrak{p} \preceq \mathfrak{p}+\boldsymbol{c} \Leftrightarrow c_{1} \geq 0 .
$$

Similarly we see that we always have $\mathfrak{p}+\boldsymbol{c} \preceq \mathfrak{p}$. Therefore

$$
\mathfrak{p} \sim \mathfrak{p}+\boldsymbol{c} \Leftrightarrow c_{1} \geq 0
$$

Set

$$
{ }^{L} L(\mathfrak{p})_{\boldsymbol{c}}= \begin{cases}t^{c} \mathbb{I}(\Omega(\boldsymbol{c})) / t^{c}(\mathbb{I}(\Omega(\boldsymbol{c})) \cap \mathfrak{p}) & \left(c_{1} \geq 0\right) \\ 0 & \text { (otherwise) } .\end{cases}
$$

Then ${ }^{L} L(\mathfrak{p})=D /\left(D s_{1}+\sum_{c_{1}<0} D_{\boldsymbol{c}}\right)=D / D \partial_{1}$ is 1-critical.

Theorem 7.7. Let $M$ be a $\delta$-critical $\mathbb{Z}^{d}$-graded left (resp. right) $D\left(R_{A}\right)$-module generated by one element $v \in M_{\mathbf{0}}$ with $\operatorname{Ann}_{F[s]}(v)=\mathfrak{p}$. Then $M$ is isomorphic to ${ }^{L} L(\mathfrak{p})$ (resp. $\left.{ }^{R} L(\mathfrak{p})\right)$.

Proof. Let $K=\operatorname{Ann}_{D\left(R_{A}\right)}(v)$. Hence $M \simeq D\left(R_{A}\right) / K$.

First we prove that $K \subseteq{ }^{L} I(\mathfrak{p})$. Let $\mathfrak{p}+\boldsymbol{a} \not \mathcal{p}$. Then $K_{\boldsymbol{a}}={ }^{L} I(\mathfrak{p})_{\boldsymbol{a}}=D\left(R_{A}\right)_{\boldsymbol{a}}$. Let $\mathfrak{p}+\boldsymbol{a} \sim \mathfrak{p}$. Let $P=t^{\boldsymbol{a}} p(s) \in D\left(R_{A}\right)_{\boldsymbol{a}}$ annihilate $v$. Suppose that $p \notin \mathfrak{p}$. Then, since $\mathfrak{p}+\boldsymbol{a} \sim \mathfrak{p}$, by the proof of Lemma 5.2 there exists $Q \in D\left(R_{A}\right)_{-\boldsymbol{a}}$ such that $v=Q P v$ as elements in $\left(M_{\mathbf{0}}\right)_{\mathfrak{p}}$. This is a contradiction; $v$ is not zero, while $P$ annihilate $v$. Hence $p \in \mathfrak{p}$. Thus we have proved the inclusion $K \subseteq{ }^{L} I(\mathfrak{p})$.

Next suppose that $K \subsetneq{ }^{L} I(\mathfrak{p})$. Let $x \in{ }^{L} I(\mathfrak{p})_{\boldsymbol{a}} \backslash K_{\boldsymbol{a}}$, and $\bar{x}$ its image in $D\left(R_{A}\right) / K \simeq M$. Since $M$ is $\delta$-critical,

$$
\delta=\operatorname{K\operatorname {dim}}{ }^{L} L(\mathfrak{p}) \leq \mathrm{K} \operatorname{dim} M / D\left(R_{A}\right) \bar{x}<\mathrm{K} \operatorname{dim} M=\delta .
$$

Hence we conclude that $K={ }^{L} I(\mathfrak{p})$. 


\title{
REFERENCES
}

[Eisenbud, D. (1995)] Commutative algebra with a view toward algebraic geometry, Grad. Texts in Math. 150, Springer-Verlag, New York.

[Gabriel, P. (1962)] Des catégories abéliennes, Bull. Soc. Math. France 90:323-448.

[Goodearl, K. R., Warfield, R. B. (1989)] An introduction to noncommutative Noetherian rings, London Math. Soc. Student Texts 16, Cambridge Univ. Press, Cambridge.

[Goldie, A. W. (1972)] Properties of the idealiser, in Ring Theory (R. Gordon, ed.), Academic Press, New York, pp. 161-169.

[Hart, R. (1971)] Krull dimension and global dimension of simple Ore-extensions, Math. Zeitschrift 121:341-345.

[Lenagan, T. H. (2000)] Dimension theory of Noetherian rings, in Infinite length modules (H. Krause, C. Ringel, ed., Bielefeld, 1998), Trends in Math., Birkhäuser, Basel, pp. 129-148.

[McConnel, J. C., Robson, J. C. (1987)] Noncommutative Noetherian rings, John Wiley \& Sons, Chichester.

[Musson, I. M. (1987)] Rings of differential operators on invariant rings of tori, Trans. of AMS 303:805827.

[Musson, I. M., Van den Bergh, M. (1998)] Invariants under tori of rings of differential operators and related topics, Mem. Amer. Math. Soc. 650.

[Rentschler, R., Gabriel, P. (1967)] Sur la dimension des anneaux et ensembles ordonnés, C. R. Acad. Sci. Paris, Sér. A 265:712-715.

[Saito, M. (2001)] Isomorphism classes of A-hypergeometric systems, Compos. Math. 128:323-338.

[Saito, M. (2007)] Primitive ideals of the ring of differential operators on an affine toric variety, Tohoku Math. J. 59:119-144.

[Saito, M., Takahashi, K. (2008)] Noetherian properties of rings of differential operators of affine semigroup algebras, to appear in Osaka J. Math.

[Saito, M., Traves, W. N. (2001)] Differential algebras on semigroup algebras, AMS Contem. Math. 286:207-226.

\author{
Department of Mathematics \\ Graduate School of Science \\ HOKKAIDO UNIVERSITY \\ SAPPORO, 060-0810 JAPAN \\ E-mail address: saito@math.sci.hokudai.ac.jp
}

